\title{
Localization Algorithm for a mobile robot using iGS
}

\author{
DaeGeun Seo *, JangMyung Lee** \\ * Electrical Engineering Department, Pusan National University, \\ Pusan, Korea, (e-mail: sdk@pusan.ac.kr). \\ ** Electrical Engineering Department, Pusan National University, \\ Pusan, Korea, (e-mail: jmlee@pusan.ac.kr)\}
}

\begin{abstract}
As an absolute positioning system, iGS is designed based on ultrasonic signals whose speed can be formulated clearly in terms of time and room temperature, which is utilized for mobile robot localization. The iGS is composed of an RFID receiver and an ultra-sonic transmitter, where an RFID is designated to synchronize the transmitter and receiver of the ultrasonic signal. The traveling time of the ultrasonic signal has been used to calculate the distance between the iGS system and a beacon which is located at a pre-specified location. This paper suggests an effective operation method of iGS to estimate position of the mobile robot working in an unstructured environment. To expand recognition range and to improve accuracy of the system, two strategies are proposed: auto-calibration of beacons belonging to neighboring blocks and removal of the environment-reflected ultrasonic signals. As the results, the ubiquitous localization system based on the iGS as a pseudo-satellite system has been developed successfully with a low cost, a high update rate, and relatively high precision.
\end{abstract}

\section{INTRODUCTION}

Recently ubiquitous environment has been applicable for the home and factory automation. As one of the typical applications, autonomous navigation system is being developed with various sensors for recognizing the surrounding environment. For the autonomous navigation, the position of the navigation system must be recognized precisely and quickly (S.M.LEE et.al., 2001). There are several indoor position recognition systems such as IR sensor based system, ultrasonic system, and camera based system. Among the various sensors system, ultrasonic sensor system is widely used since the system is cheap and simple to implement while it is neither applicable for long distance measuring nor robust against the reflected waves (H.Premans et.al., 1993). There are several location recognition systems using ultrasonic sensors such as Active Bat, iGS, etc. There have been several researches on this research where three or more beacons in a small room are utilized for the location recognition (A.M Flynn, 1998 and Tatsuo Arail et.al., 1983). However currently for public services, the mobiles are required to move in a wider space. The task is not feasible using the ultrasonic sensors which are sensitive to noises and have a short effective distance. Therefore in this paper to apply iGS not in a small room but in a wider service area, a block expansion algorithm is introduced. When the mobile robot is moving to a new block, a new set of beacons needs to be selected to get the position data more precisely instead of keeping the same set of beacons. Selecting the new set of beacons, eliminating reflected signal effects against obstacles, and reducing the environment noise effects are major problems to be solved for the robust control of mobile robot in a wider area.
Section 2 introduces basic principles of indoor location recognition using ultrasonic sensors and iGS which are used for real experiments. Section 3 proposes a new auto calibration algorithm to recognize the beacon coordinates when the mobile robot is moving into a new block, which reduces the localization time and improves the data reliability. Section 4 proposes NRW algorithm to resolve the sensitiveness of ultrasonic sensors to environment noise and reflected wave. Section 5 verifies effectiveness of the proposed algorithms through the real experiments.

\section{Location Measurement}

\subsection{Distance measurement}

Basically the distance measurement can be done by measuring traveling time of the ultrasonic signal and its velocity. The ultrasonic wave TOF (traveling Time Of Flight) can be usually detected by using the threshold value as shown in (Fig. 1) for the received signal. The transmission request signal is sent out from the transmitter at $\mathrm{T}_{\mathrm{t}}$, and the ultrasonic signal is received at $T_{r}$. The difference between the two represents the traveling time of the ultrasonic signal from a beacon to the mobile robot (S.Hong et.al., 2004).

$$
T O F=T_{r}-T_{t}
$$

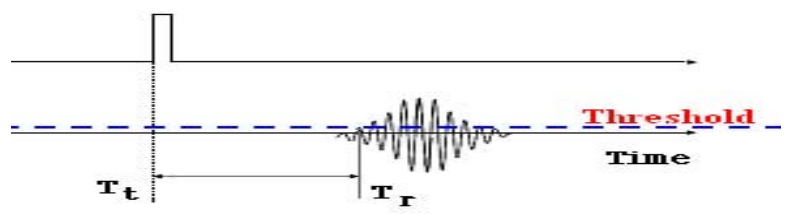

Fig. 1. Time Of Flight 
The velocity of the ultrasonic wave, $c$, is dependent on the absolute temperature, $T$, and it is represented as

$$
c \cong 331.5 \sqrt{\frac{T}{237}}[\mathrm{~m} / \mathrm{s}]
$$

Even though the velocity may depend on other factors of the transmission channel, "(2)," is a general form for ultrasonic waver velocity (Billur Barshan, 2000 and M.Parrialla et.al., 1991).

In this research, the working environment of the mobile robot is kept at room temperature of $24^{\circ} \mathrm{C}$ normally. Therefore the velocity, $c$, is replaced by $343 \mathrm{~m} / \mathrm{s}$ to speed up the measurement process. And the distance, $d$, from the mobile robot to a specific beacon can be calculated as the multiplication of the velocity and the traveling time as

$$
d=c \times T O F
$$

\section{2 iGS(indoor GPS system)}

As an indoor localization system, iGS has been used in this research, which is developed by PNU Intelligent Robot Lab. and Ninety Systems Corp. As illustrated in (Fig. 2), a localizer which is installed on the mobile robot sends out an RF ID signal to call a beacon. When a corresponding beacon receives the RFID signal, it sends out the ultrasonic wave signal to the localizer. The TOF can be determined by counting the time from the moment of RFID transmission to the moment of ultrasonic wave arrival at the localizer. Since the localizer is located at a point in three dimensional space, if there are three distant data from different beacons at a moment, the three dimensional position coordinates can be calculated by the triangulation technique. From the general triangulation technique illustrated in (Fig. 3), the absolute coordinates of the mobile robot-the localizer-- can be determined uniquely.

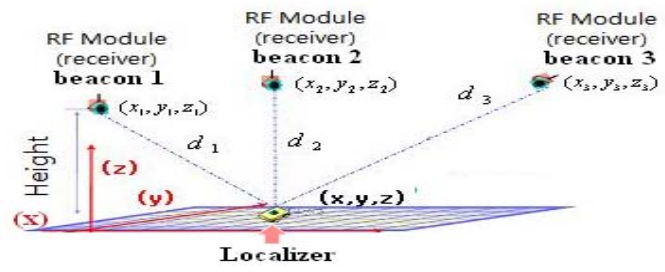

Fig. 2. Composition of robot localization system.

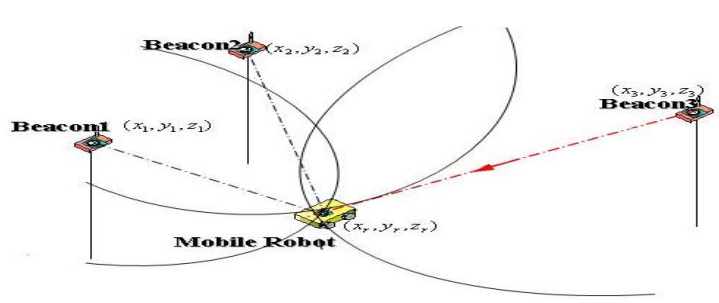

Fig. 3. General triangulation technique.

(Fig. 4) illustrates communication flow between iGS and the localizer of iGS. To extend battery lifetime of beacons, the sleep mode is utilized until the corresponding RFID comes into the beacon. EKF (Extended Kalman Filter) is utilized to improve the localization accuracy (S.B. Kimetal.,2007).

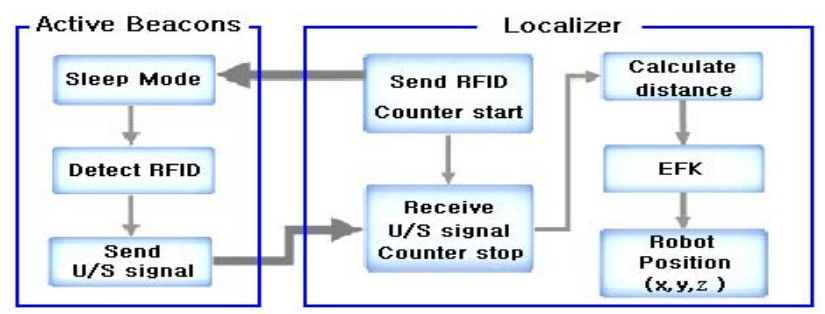

Fig. 4. Operating sequence of the iGS and localizer.

When the localizer sends out a RFID signal, the counter starts to count, and it stops when it receives the ultrasonic signal from the corresponding beacon. Ignoring the traveling time of RFID, the traveling time of the ultrasonic signal can be measured by the counter. Using the counter value and the traveling speed which is deterministic, the distance from the localizer to the corresponding beacon can be calculated as explained previously. Based on the distance values to the beacons which are holding their own position data, the location of the mobile robot can be calculated.

When the distances from the localizer to the beacons are represented as $d_{1}, d_{2}$ and $d_{3}$, and the absolute positions of the beacons are $\left(x_{1}, y_{1}, z_{1}\right),\left(x_{2}, y_{2}, z_{2}\right)$ and $\left(x_{3}, y_{3}, z_{3}\right)$, the location coordinates of the mobile robot, $\left(x_{r}, y_{r}, z_{r}\right)$, can be obtained as an intersection of three circles illustrated in (Fig. 3 ). From the circle equations, the mobile robot coordinates can be calculated as follows:

$$
\left[\begin{array}{c}
\left(x_{r}-x_{1}\right)^{2}+\left(y_{r}-y_{1}\right)^{2}+\left(z_{r}-z_{1}\right)^{2} \\
\left(x_{r}-x_{2}\right)^{2}+\left(y_{r}-y_{2}\right)^{2}+\left(z_{r}-z_{2}\right)^{2} \\
\left(x_{r}-x_{3}\right)^{2}+\left(y_{r}-y_{3}\right)^{2}+\left(z_{r}-z_{3}\right)^{2}
\end{array}\right]=\left[\begin{array}{c}
d_{1}^{2} \\
d_{2}^{2} \\
d_{3}^{2}
\end{array}\right] .
$$

For the beacon 1, “(4),” can be expanded.

$$
d_{1}^{2}=x_{r}^{2}+y_{r}^{2}+z_{r}^{2}-2 x_{r} x_{1}-2 y_{r} y_{1}-2 z_{r} z_{1}+x_{1}^{2}+y_{1}^{2}+z_{1}^{2} .
$$

This form of three equations for beacon 1, 2 and 3 can be reordered for unknown mobile robot coordinates, $\left(x_{r}, y_{r}, z_{r}\right)$

$$
\left[\begin{array}{lll}
2 x_{1} & 2 y_{1} & 2 z_{1} \\
2 x_{2} & 2 y_{2} & 2 z_{2} \\
2 x_{3} & 2 y_{3} & 2 z_{3}
\end{array}\right]\left[\begin{array}{l}
x_{r} \\
y_{r} \\
z_{r}
\end{array}\right]=\left[\begin{array}{l}
x_{1}^{2}+y_{1}^{2}+z_{1}^{2}+x_{r}^{2}+y_{r}^{2}+z_{r}^{2}-d_{1}^{2} \\
x_{2}^{2}+y_{2}^{2}+z_{2}^{2}+x_{r}^{2}+y_{r}^{2}+z_{r}^{2}-d_{2}^{2} \\
x_{3}^{2}+y_{3}^{2}+z_{3}^{2}+x_{r}^{2}+y_{r}^{2}+z_{r}^{2}-d_{3}^{2}
\end{array}\right] .
$$

Now the unknown mobile robot coordinates can be calculated as

$$
X=A^{-1} B
$$

where

$$
X=\left[\begin{array}{l}
x_{r} \\
y_{r} \\
z_{r}
\end{array}\right] \quad, A=\left[\begin{array}{lll}
2 x_{1} & 2 y_{1} & 2 z_{1} \\
2 x_{2} & 2 y_{2} & 2 z_{2} \\
2 x_{3} & 2 y_{3} & 2 z_{3}
\end{array}\right]
$$


and

$$
B=\left[\begin{array}{l}
x_{1}^{2}+y_{1}^{2}+z_{1}^{2}+x_{r}^{2}+y_{r}^{2}+z_{r}^{2}-d_{1}^{2} \\
x_{2}^{2}+y_{2}^{2}+z_{2}^{2}+x_{r}^{2}+y_{r}^{2}+z_{r}^{2}-d_{2}^{2} \\
x_{3}^{2}+y_{3}^{2}+z_{3}^{2}+x_{r}^{2}+y_{r}^{2}+z_{r}^{2}-d_{3}^{2}
\end{array}\right] .
$$

3. Multi Block Scheme with Auto Calibration

\subsection{Auto Calibration Algorithm}

For the absolute localization process, the beacons are installed on the pre-determined position of ceiling or walls. However it is very difficult to attach the beacon precisely at a specific position since installing beacons on the ceiling and walls is not an easy task. To remove out this hard and erroneous installation process of the beacons and to obtain reliable absolute position data, an auto calibration algorithm has been developed (S.B.Kim et.al, 2006a). With this auto calibration algorithm, the location of the mobile robot can be estimated quickly and precisely when it is moving out and into a new block as illustrated in (Fig. 5) (S.B.Kim et.al, 2006b).

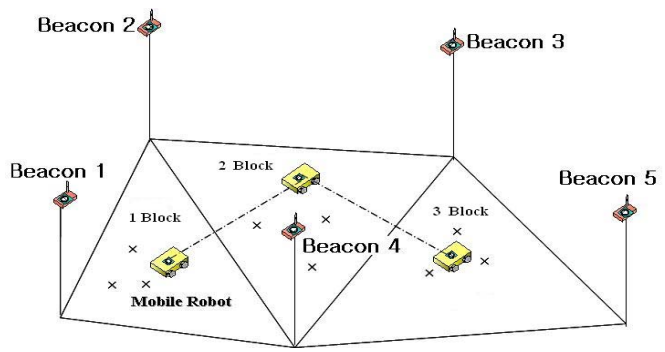

Fig. 5. Block expanding using auto calibration

There are three blocks each of which is composed of three beacons for the block expanding experiments. Every 5,000 $\mathrm{mm}$, a beacon is installed at the top of a poll in (Fig. 5). At the initial stage of the experiment, the mobile robot goes through the auto calibration process by locating the mobile robot on the three $\mathrm{x}$ marked positions whose absolute coordinates are precisely specified. With the process, the mobile robot has its own position coordinates. When the mobile robot is moving into the block 2 , coordinates of the new beacon 3 can be calculated through the auto calibration process using the current location information of the mobile robot and the absolute position information of beacons 2 and 4. This form of three equations for beacon 2, 4 and 3 can be reordered for unknown mobile robot coordinates, $\left(x_{r}, y_{r}, z_{r}\right)$.

$$
\left[\begin{array}{lll}
2 x_{2} & 2 y_{2} & 2 z_{2} \\
2 x_{3} & 2 y_{3} & 2 z_{3} \\
2 x_{4} & 2 y_{4} & 2 z_{4}
\end{array}\right]\left[\begin{array}{l}
x_{r} \\
y_{r} \\
z_{r}
\end{array}\right]=\left[\begin{array}{l}
x_{2}^{2}+y_{2}^{2}+z_{2}^{2}+x_{r}^{2}+y_{r}^{2}+z_{r}^{2}-d_{2}^{2} \\
x_{3}^{2}+y_{3}^{2}+z_{3}^{2}+x_{r}^{2}+y_{r}^{2}+z_{r}^{2}-d_{3}^{2} \\
x_{4}^{2}+y_{4}^{2}+z_{4}^{2}+x_{r}^{2}+y_{r}^{2}+z_{r}^{2}-d_{4}^{2}
\end{array}\right]
$$

Now the unknown mobile robot coordinates can be calculated as

$$
X=\left[\begin{array}{l}
x_{r} \\
y_{r} \\
z_{r}
\end{array}\right] \quad, A=\left[\begin{array}{lll}
2 x_{2} & 2 y_{2} & 2 z_{2} \\
2 x_{3} & 2 y_{3} & 2 z_{3} \\
2 x_{4} & 2 y_{4} & 2 z_{4}
\end{array}\right]
$$

and

$$
B=\left[\begin{array}{l}
x_{2}^{2}+y_{2}^{2}+z_{2}^{2}+x_{r}^{2}+y_{r}^{2}+z_{r}^{2}-d_{2}^{2} \\
x_{3}^{2}+y_{3}^{2}+z_{3}^{2}+x_{r}^{2}+y_{r}^{2}+z_{r}^{2}-d_{3}^{2} \\
x_{4}^{2}+y_{4}^{2}+z_{4}^{2}+x_{r}^{2}+y_{r}^{2}+z_{r}^{2}-d_{4}^{2}
\end{array}\right] \text {. }
$$

While the mobile robot is moving in the block 2 , the position of the mobile robot is updated by the three beacon data. When it moves into block 3, the coordinates of beacon 5 is obtained by the same auto calibration process. By using this block expansion and auto calibration algorithms, a mobile robot can move around wider area with reliable and speedy position recognition.

\subsection{Block expansion}

Another shortage of using the ultrasonic sensor for the indoor localization is that the ultrasonic signal attenuates quickly and becomes unreliable when it is traveling in the noisy environment. Since the maximum traveling distance for the ultrasonic signal of iGS is $15 \mathrm{M}$, the block expansion technique using multiple beacons is essential for public service areas such as airport and train stations. For these applications, as an examples the wide area is divided into 9 blocks or cells in mobile communication system in (Fig. 6). The block number is necessary to be minimized for cost effectiveness while it is large enough to provide accuracy and reliability.

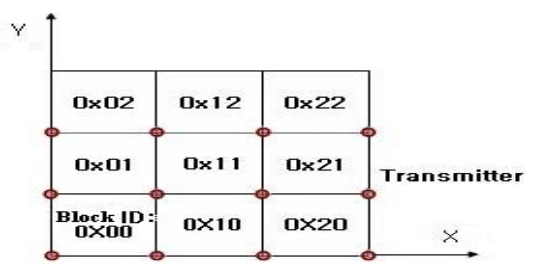

Fig. 6. Structure of block expansion

\begin{tabular}{|c|c|c|c|c|c|c|c|c|}
\hline \multicolumn{3}{|c|}{ Number of Transmitter } & \multicolumn{3}{|c|}{$\mathrm{X}$ axis of Block } & \multicolumn{3}{|c|}{$Y$ axis of Block } \\
\hline Bit8 & $\therefore$ Bitz" & Bit6 & Bit5 & Bit4 4. & $\because$ Bit3 & Bit2 & Bit1 & B̈ito. \\
\hline$\because, 0 / 1, \cdots$ & $\theta_{*}, \mathrm{xa}_{3} \ldots$ & $\times 2$ & $x 1$ & $\ldots \times 0 \ldots$ & $\sqrt{3} \ldots$ & $Y 2$ & Y1 & ....Y.... \\
\hline $\begin{array}{l}\text { Trans } \\
\text { mitter }\end{array}$ & \multicolumn{4}{|c|}{ data of Block } & \multicolumn{4}{|c|}{ data of Block } \\
\hline
\end{tabular}

For the given multiple blocks, an ID needs to be assigned uniquely to each individual block, which is illustrated in (Fig. 7).

Fig. 7. ID format of beacons

The beacon ID's in the same block are defined to have close codes to distinguish from the beacon ID in a new block easily. The overall flowchart of block expansion algorithm is shown in (Fig. 8) where a new beacon is coming to be used for the localization when the mobile robot is moving into a new block. 


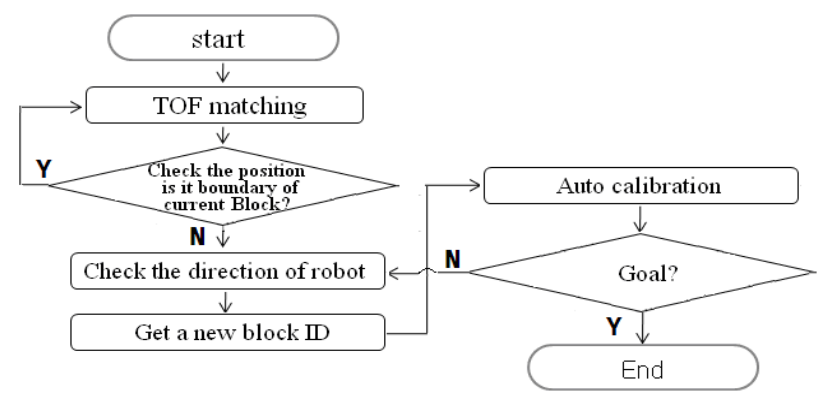

Fig. 8. Flowchart of block expansion algorithm

The next block for the mobile robot is estimated from the velocity vector of the mobile robot, which shortens the auto calibration time. For the optimal auto calibration, three beacons are assigned to each block. If there exists obstacles which prevent the sonic signal traveling from a beacon to the localizer while the mobile robot is moving in a block identification, the Flat Floor Algorithm(S.B.Kim et.al, 2006b) for missing one beacon data and Linear Incremental Algorithm(S.B.Kim et.al, 2006a) for missing two beacon data have been applied for the temporary localization. For the maximum sixteen blocks along $\mathrm{x}$-axis and $\mathrm{y}$-axis, respectively, a four bits data frame has been assigned. Therefore the mobile robot can move around the maximum 256 blocks. When a block size is $5 \mathrm{~m} \times 5 \mathrm{~m}$, the mobile robot can work in $80 \mathrm{~m} \times 80 \mathrm{~m}$ space recognizing its own position precisely.

\section{Random Noise Filtering}

\subsection{Error from Environment Noise}

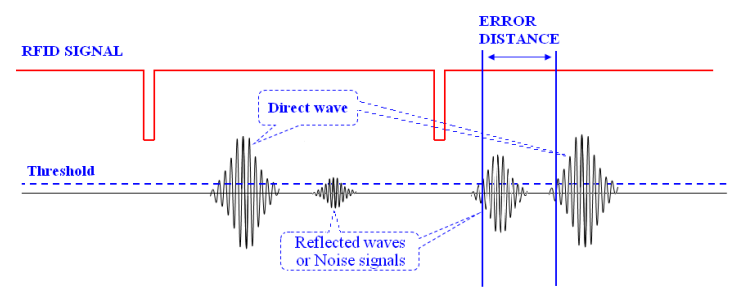

Fig. 9. Effect of noise

The ultrasonic signal generated for the previous RFID can be detected as a signal before the current signal is coming. Or the noise signals may arrive at the receiver as a signal as illustrated in (Fig. 9). To remove out these malfunctioning situations, the gap between two RFID signals can be extended and the threshold value can be increased not to receive the small noises as signals. However these treatises limit the working space and deteriorate the data acquisition reliability by lengthening the data processing time.

\subsection{NRW Algorithm}

An NRW (Noise Rejection Window) algorithm is proposed in this paper to overcome the malfunctioning situations where the direct signals are hided behind the relatively large noise signals. The noise signals can be generated by the reflection of the previous signal to some obstacles located in the signal path. Or it can be generated by other environmental sources like music and construction sound. The mobile robot needs to be robust against surrounding obstacles and environment, which is the final goal of NRW algorithm. In the algorithm, the previous flight distance data of the ultrasonic signal have been utilized for estimating next flight distance.

$$
\begin{aligned}
& \begin{array}{|l|}
\hline d_{11}, d_{21}, d_{31} \\
\hline
\end{array} \\
& t_{1} d_{12,} d_{22}, d_{32} \\
& t_{2} \frac{d_{13,} d_{23}, d_{33}}{t_{3} d_{14,} d_{24}, d_{34}} \\
& t_{n} d_{1 n} d_{2 n,} d_{3 n}
\end{aligned}
$$

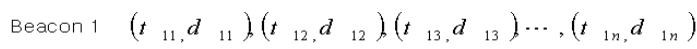

Fig. 10. Windows data of $\mathrm{i}^{\text {th }}$ beacon

The difference between two neighboring flight distance is small enough to be within the tolerance error of iGS, $5 \mathrm{Cm}$, when the mobile robot is moving not so fast, which provides the flight distance range of ultrasonic signal for each step. The flight distance data in the pre-specified window can be utilized to estimate the next step flight distance, since the mobile robot motion is not abruptly changing. (Fig 10) illustrates the data format of flight distance stored in the window, where $d_{B i j}$ is received $\mathrm{n}^{\text {th }}$ flight distance data, $i$ is the beacon identification number, $j$ is the sequence number of beacon which is limited to as twenty considering the data processing speed. When $d_{i j}$ is measured, the value is checked whether that is reliable or not by NRW algorithm as

$$
\begin{aligned}
& \text { tolerance }>\left|d_{i(j-1)}-d_{i j}\right|, \quad \bar{d}_{i j}=d_{i j} \\
& \text { otherwise } \quad, \bar{d}_{i j}=\hat{d}_{i j}
\end{aligned}
$$

where $\bar{d}_{i j}$ is the result of NRW algorithm. The estimated flight distance of beacon $i$ at the moment of $j, \widehat{d}_{i j}$ can be determined as

$$
\begin{gathered}
\hat{d}_{i j}=d_{i(j-1)}+\sigma_{i} \\
\sigma_{i}=\sqrt{\frac{1}{w} \sum_{j=n-w}^{n-1} x_{i j}^{2}-m^{2}}
\end{gathered}
$$

where $\sigma_{i}$ is standard deviation of the data stored in the window, $w$ is the window size, $x_{i j}$ is the variance of the data in the window, $m$ is the average of the data stored in the window, $d_{i(j-n)}$ is the $\mathrm{n}$ step previous flight distance data of the $i^{\text {th }}$ beacon .To obtain the estimated value, standard deviation of the flight distance data stored in the window for each beacon has been obtained. As shown in "(13),", the value is added to the previous window data for the beacon to estimate $\hat{d}_{i j}$. 


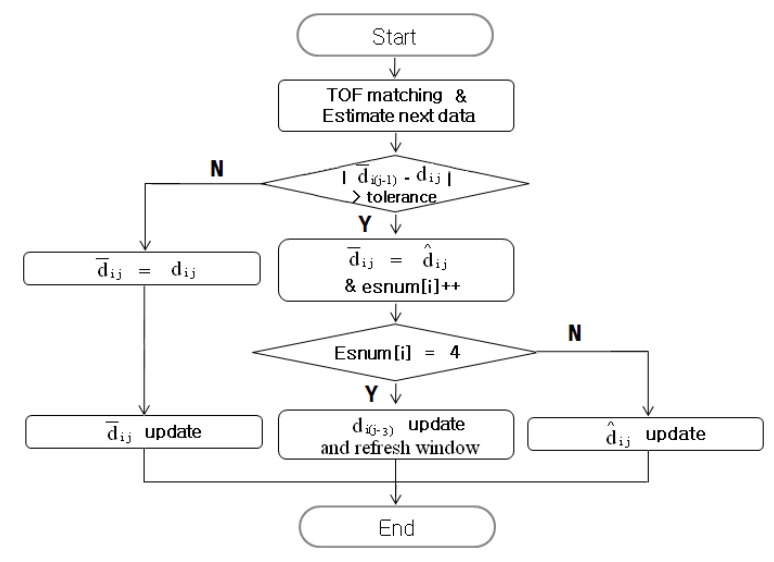

Fig. 11. Flowchart of NRW algorithm

(Fig. 11) is flowchart for NRW algorithm, where the localizer receives ultrasonic signals from beacons sequentially. Using the ultrasonic flight time, the flight distance is calculated. With the calculated distance, the filtering process checks whether the data exist within the tolerance bound or not. Unless the data exist within the tolerance bound, it is considered as a malicious signal affected by obstacles or environmental noises. In this case, the current flight distance is estimated as $\hat{d}_{B i j}$, the summation of the previous flight distance and the standard deviation of the flight distance in the window as shown in "(13),". In the other case, the flight distance is assumed to be reliable and it is selected as $\bar{d}_{i j}$, the updated flight distance for the current step. When the estimated values of $\hat{d}_{B i j}$ are used continuously, the absolute error becomes large and large. This situation is handled by refreshing the window when the estimated values of $\hat{d}_{i j}$ are used more than four times consecutively. The number four is heuristically and empirically determined with the window size of twenty.

\section{Experiments and Results}

For indoor localization of the mobile robot to verify the effectiveness of the newly proposed NRW algorithm, a mobile robot is designed with the installation of iGS. The iGS consists of localizer and beacon. The localizer which is carried by the mobile robot sends out RFID signals and receives ultrasonic signals to measure the flight time of the ultrasonic signal; the beacon receives the RFID signal and checks with its own ID to send out the ultrasonic signal when the two codes match to each other. In the localizer, rfPIC12F675F is used to control the RFID, TMS320C2406A is used for system control, and AR40-10P(40kHz)is used to receive the ultrasonic signal. In the beacon, MSP403F1101A is used for the system control, and AT40-10P $(40 \mathrm{kHz})$ is used for the ultrasonic transmission. Noise sources are placed in the experimental environment without any specific ID. The mobile robot consists of driving part and control part. For the driving part, 4S56Q-03554S stepping motors are utilized to move and PIC18F8720 is used to control the motors. The maximum velocity of the mobile robot is kept as $35 \mathrm{~cm} / \mathrm{s}$ not to cause dangerous situations to human. For the experimental environment in (Fig. 12), the active beacons are installed for location recognition and one additional beacon is placed to generate noisy signals randomly. For the auto calibration process, the mobile robot is placed at the pre-determined locations, $\mathrm{A}(100,101), \mathrm{B}(750,201)$, and $\mathrm{C}(500,301)$ in a sequence. With the process, the coordinates of beacons are obtained as beacon 1 (-215,-673,1795), beacon 2 $(3752,1037,1501)$, beacon $3(-293,1836,1888)$. The noise source is located at $(2600,800,1800)$.

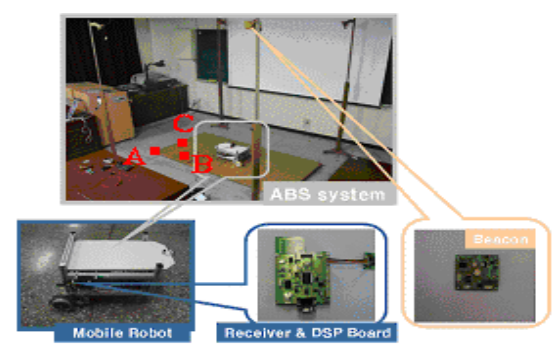

Fig. 12. Experimental environment

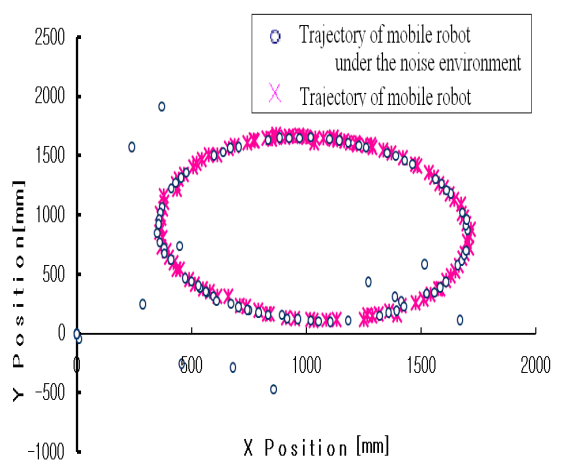

Fig. 13. Trajectory of robot under the noise environment

The noise characteristics should be random and unexpectable. However the continuous noises which have the same frequency as the beacon cannot be filtered out. Therefore in this experiment the noise source is activated every $200 \mathrm{~ms}$ while the mobile robot is moving along a circular path as shown in (Fig. 13). The small circles in (Fig. 13) represent the measured locations of the mobile robot while the mobile robot is moving on a circular path marked as X. By the noise interferences, the localization process is not successful at all.

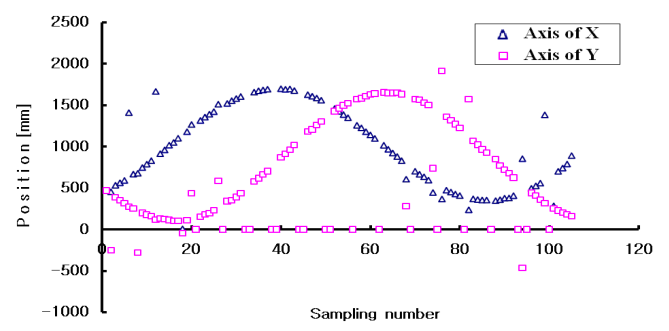

Fig. 14. Without NRW algorithm 
(Fig. 14) represents the measured trajectory of the mobile robot along $\mathrm{X}$-axis and $\mathrm{Y}$-axis respectively, while it is moving on a circle under noisy environment.

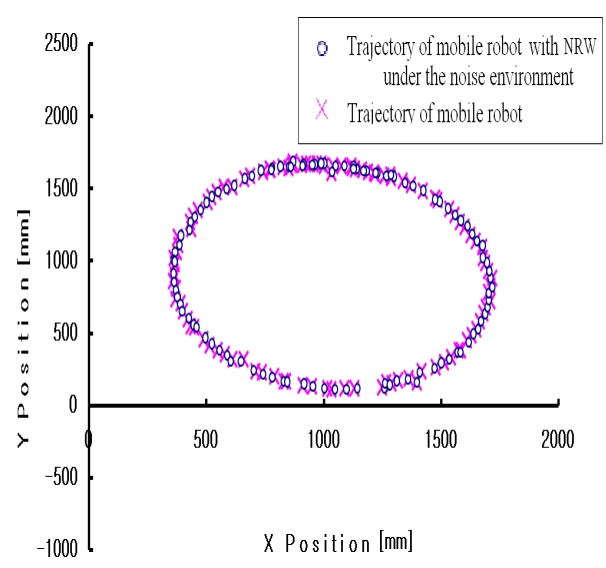

Fig. 15. Trajectory of robot with NRW under the noise environment

To show the effectiveness of NRW algorithm, the same experiment is performed by applying NRW algorithm for the circular motion of the mobile robot. The results are illustrated in (Fig. 15). It is clear that with NRW algorithm, the mobile robot carrying iGS recognizes real locations precisely while there are several interferences of ultrasonic noise sources.

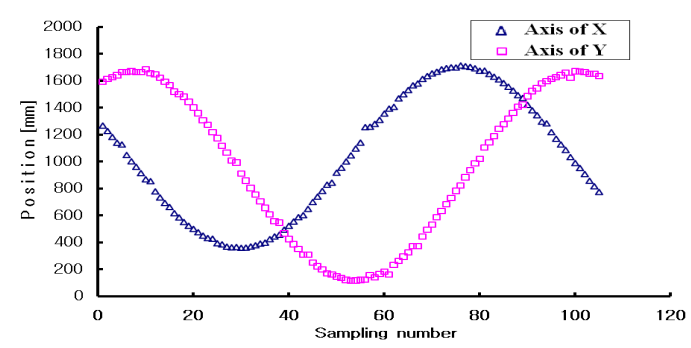

Fig. 16. With NRW algorithm

(Fig. 16) represents the robot trajectory in terms of $\mathrm{X}$-axis and $\mathrm{Y}$-axis respectively, while there exist ultrasonic noise sources.

\section{Conclusions}

To build ubiquitous environment for a mobile robot moving inside buildings or underground where GPS signals cannot get into clearly, iGS using ultrasonic sensors provides a localization system which is cheap, has fast data update rate, and provides high position accuracy. For the outdoor environment, GPS is popular. However the ultrasonic signals are very sensitive to noises. Therefore ultrasonic sensors are malicious when there are some obstacles in the signal path or when the environment itself is noisy. This paper proposes a block expansion algorithm with an auto calibration and NRW algorithms to overcome these shortcomings in using ultrasonic sensors and to provide a cheap and robust localization system for a mobile robot moving in a wider indoor environment. The auto calibration algorithm shortens localization time and improves the data reliability when the mobile robot moves into a new block. The NRW algorithm provides a robust localization scheme to eliminate the noises caused by the obstacles in the ultrasonic signal path as well as the active environment itself. Effectiveness of the proposed algorithm is demonstrated through the real experiments.

\section{Acknowledgements}

"This work was partly supported by the IT R\&D program of MIC/IITA[2005-S-111-02, intelligent robot sensor] and Ninety System co., Ltd".

\section{REFERENCES}

San Mok Lee (2001). Using Vision Sensor and Sonar Sensor of Mobile Robot Localization. Chungnam National University.

H. Premans and V. Campenhout (1993). High Resolution Sensor Based on Tri-aural Perception. IEEE Trans. On Robotics and Automation, vol. 9, no.1, pp. 3648.

A. M. Flynn (1998). Combining Sonar and Infrared Sensors for Mobile Robot Navigation. The International Journal of Robot Research, vol. 7, no.6, pp. 5-14.

Tatsuo Arail, and Eiji Nakatno (1983). Development of Measuring Equipment for Location and Direction Using Ultrasonic Waves. ASME Trans., vol. 105, pp. 152156.

S. Hong, M. H. Lee, S. H. Kwon, and H. H. Chun (2004). A Cat Test for the Estimation of GPS/INS Alignment Errors. IEEE Trans. Intelligent Transportation Systems, vol. 5 , no.3.

Billur Barshan (2000). Fast Processing Techniques for Accurate Ultrasonic Range Measurements. IOP J. Meas. Sci. Technolog, vol. 11, pp. 45-50.

M. Parrialla, J. J. Anaya and C. Fritsch (1991). Digital Signal Processing Techniques for High Accuracy Ultrasonic Range Measurements. IEEE Trans. Instrumentation and Measurement, vol. 40, no. 4, pp. 759-763.

S. B. Kim and JangMyung Lee (2007). Mobile Robot Localization Using Auto Calibration Algorithm. KRS Journal, vol. 2, no. 1, pp. 40-47.

S. B. Kim and J. M. Lee (2006). Robust Positioning a Mobile Robot with Active Beacon Sensors. LNAI 425, ISSN 0302-9743, Part 1, pp. 890-897.

S. B. Kim, I. O. Lee and J. M. Lee (2006). Dynamic Localization of a Mobile Robot with Active Beacon Sensors. 4th IFAC Symposium on Mechatronic Systems Heidelberg Germany, Sept. 12-14 2006.

Iowa State University GPS page Web site at http://www.cnde.iastate.edu/gps.htm1. 